\title{
Selective use of percutaneous testis biopsy to optimize IVF-ICSI outcomes: a case series
}

\author{
Russell P. Hayden ${ }^{1}$, Diane L. Wright ${ }^{2}$, Thomas L. Toth ${ }^{3,4}$ and Cigdem Tanrikut ${ }^{1,4^{*}}$
}

\begin{abstract}
Background: Sperm quality may degrade during transit through the male reproductive tract in some individuals. In this setting surgically retrieved testicular sperm may outperform ejaculated samples for use with in vitro fertilization (IVF) and intracytoplasmic sperm injection (IVF-ICSI). We sought to describe one center's experience with the use of fresh testicular sperm after prior failed IVF-ICSI with ejaculated samples.

Results: A retrospective review was conducted evaluating IVF-ICSI cycles performed at a tertiary IVF unit between 2009 and 2014. Couples who were managed with percutaneous testis biopsy to obtain sperm, despite availability of ejaculated sperm, were included. Four couples who underwent a total of 6 percutaneous testis biopsy/IVF-ICSI cycles were identified. Collectively, the couples had undergone 9 prior IVF-ICSI cycles using fresh ejaculated sperm without successful pregnancy. From the six cycles that used fresh testicular sperm four live births resulted (1 twin gestation, 3 singletons). Only 1 of the 4 couples remained childless.

Conclusions: For patients who have had prior failed IVF-ICSI attempts, this small case series demonstrates a possible therapeutic benefit when freshly procured testicular sperm are used in lieu of ejaculated samples.
\end{abstract}

Keywords: IVF, ICSI, Male factor infertility, TESE, Kartagener syndrome

\section{Background}

With the advent of in vitro fertilization (IVF) and intracytoplasmic sperm injection (IVF-ICSI), many men with severe male factor infertility can now be offered the opportunity to parent their own genetic offspring. Unfortunately, a subset of patients will not succeed despite these advanced treatments. Although the chance of a live birth continues to rise with an increasing number of IVF cycles, up to $19 \%$ of couples will remain childless following 8 attempts [1]. Given the financial, physical, and psychological demands each IVF cycle requires [2], a significant drop-out rate accrues with each successive treatment [3].

For some couples with multiple failed IVF cycles, sperm-related factors may play a principle role. Unfortunately, robust indicators of sperm quality have yet to be identified. Most centers routinely select for sperm based upon visual cues such as motility and morphology. Fresh ejaculated sperm, when available, are generally preferred

\footnotetext{
* Correspondence: ctanrikut@mgh.harvard.edu

'Department of Urology, Massachusetts General Hospital, Boston, MA, USA

${ }^{4}$ MGH Fertility Center, 55 Fruit Street, YAW 10A, Boston, MA 02114, USA

Full list of author information is available at the end of the article
}

over surgically retrieved samples given the need for an invasive procedure. These practice patterns are often based upon the belief that immature testicular sperm may be less likely to result in a successful pregnancy. However, this tenet is increasingly being challenged as IVF-ICSI outcomes correlate poorly with traditional sperm quality parameters $[4,5]$. Studies involving large cohorts have now shown near equivalence with samples retrieved from the epididymis or testis [6-8], and even with sperm characterized by poor motility and morphology [5]. In the extreme scenario of substantial necrospermia, testicular sperm may even outperform viable ejaculated samples [9].

Investigators have begun to focus on more sophisticated rubrics of sperm quality in order to understand and optimize IVF-ICSI outcomes. Reactive oxygen species (ROS) and DNA damage have been two areas of intense investigation. Preliminary research has also shown that sperm quality, especially in terms of DNA integrity, may decrease in some patients as the spermatozoa transit through the male reproductive tract [10-12]. As a result, some couples may benefit 
from testis-derived sperm even in the presence of viable sperm in the ejaculate.

Although it is difficult to rule out an occult spermrelated etiology when evaluating a couple with recurrent IVF failure, an attempt with testicular sperm is an option rarely offered when ejaculated sperm are available. The following case series explores the premise that a subset of couples may benefit from freshly procured testicular sperm in an attempt to bypass male factors related to post-testicular events.

\section{Methods}

IRB approval was obtained for this investigation. A retrospective chart review was conducted to evaluate all IVF-ICSI cycles at a tertiary referral center. Records from January 2010 to January 2014 were examined. Inclusion criteria consisted of couples who were managed with percutaneous testis biopsy (PercBx) for any indication at our institution. A total of 19 consecutive cases were identified. Men who had obstructive azoospermia as the indication for PercBx were excluded from the study population, which resulted in four unique couples (Table 1) who underwent a total of six IVF-ICSI treatment cycles using freshly retrieved testicular sperm (Table 2). Cryopreservation was not included in our treatment protocol to avoid diminished sperm yields from the freeze-thaw cycle, and to avoid the theoretical risk of reduced implantation rates [13].

All couples had undergone at least one unsuccessful IVF-ICSI cycle using a fresh ejaculated sample (mean 2.25 cycles) prior to proceeding to IVF-ICSI with PercBx. Female partners were evaluated by a reproductive endocrinologist and optimized for another IVF-ICSI cycle. Each male partner underwent thorough urologic evaluation and informed consent prior to proceeding with percutaneous testis biopsy. Semen analyses were interpreted according to the $2010 \mathrm{WHO}$ guidelines. Normal testis volume was considered $>15 \mathrm{~mL}$. All patients underwent a basic endocrine work-up, including follicle stimulating hormone (FSH, lab normal $<12 \mathrm{mIU} / \mathrm{mL}$ ), and morning total testosterone (normal $>270 \mathrm{ng} / \mathrm{dL}$ ).
All sperm procurements were coordinated on the day of oocyte retrieval. Male evaluations and testicular biopsies were performed by a single reproductive urologist (CT). After initiating local anesthesia via a spermatic cord block an 18-gauge hollow core biopsy needle was used to percutaneously procure 4 to 10 cores of testicular parenchyma. An embryologist provided immediate feedback to confirm the presence of adequate numbers of sperm for the anticipated number of oocytes. Unilateral biopsy was sufficient in each circumstance.

\section{Results}

\section{Case 1}

The couple presented with a history of 4 failed IVF-ICSI cycles in the setting of known oligoasthenoteratospermia (OAT). Further testing revealed an elevated sperm DNA fragmentation index (DFI). The female partner was 31 years old with an unremarkable gynecologic evaluation.

Her partner was a healthy 34-year-old nonsmoker with no prior conceptions or conception attempts. On physical exam the patient was found to have symmetric $27 \mathrm{cc}$ testes. There was no palpable varicocele. His FSH was $1.2 \mathrm{mIU} / \mathrm{mL}$ and his morning testosterone was $420 \mathrm{ng} / \mathrm{dL}$. Four semen analyses were available and remarkable for variable OAT, with concentrations $0.1-27$ million/cc, progressive motility $0-25 \%$, and Kruger morphology $0-1 \%$ normal forms. Total motile counts ranged from 0.3 to 13 million. His karyotype was normal.

The couple's four prior IVF-ICSI attempts utilized ejaculated sperm. Each cycle resulted in the retrieval of 8 to 14 mature oocytes. The first cycle resulted in an early spontaneous abortion. The second attempt yielded no fertilized oocytes. Fertilization rates of the third and fourth cycles were $44 \%$ and $64 \%$ with 1 and 2 embryos transferred, respectively. Neither cycle resulted in pregnancy. Given the couple's history of 4 failed IVF-ICSI cycles a sperm chromatin structure assay (SCSA) was ordered, noting an elevated DFI of $44.2 \%$.

In light of four unsuccessful IVF-ICSI cycles and indicators of poor sperm quality, including elevated DFI, the

Table 1 Couples' clinical characteristics

\begin{tabular}{|c|c|c|c|c|c|}
\hline & \multirow{2}{*}{$\begin{array}{l}\text { Number of } \\
\text { prior cycles }\end{array}$} & \multicolumn{2}{|l|}{ Male } & \multicolumn{2}{|c|}{ Female } \\
\hline & & Age & Infertility diagnoses & Age & Infertility diagnoses \\
\hline \multirow[t]{2}{*}{ Case 1} & 4 & 34 & $\mathrm{OAT}^{\mathrm{a}}$ & 31 & None \\
\hline & & & Elevated DFl ${ }^{\mathrm{b}}$ & & \\
\hline \multirow[t]{2}{*}{ Case 2} & 1 & 49 & OAT & 37 & Advanced Maternal Age \\
\hline & & & & & Corrected Uterine Factor \\
\hline Case 3 & 3 & 32 & Kartagener's Syndrome & 32 & Corrected Uterine Factor \\
\hline \multirow[t]{2}{*}{ Case 4} & 1 & 46 & OAT & 38 & Advanced Maternal Age \\
\hline & & & Elevated DFI & & Decreased Ovarian Reserve \\
\hline
\end{tabular}

\footnotetext{
aOligoasthenoteratospermia, ${ }^{b}$ DNA fragmentation index
} 
Table 2 IVF-ICSI cycle outcomes using testis-derived sperm obtained via percutaneous biopsy

\begin{tabular}{llllll}
\hline & $\begin{array}{l}\text { Number of oocytes } \\
\text { (number mature) }\end{array}$ & $\begin{array}{l}\text { Number } \\
\text { fertilized (\%) }\end{array}$ & $\begin{array}{l}\text { Number of 6-10 cell } \\
\text { embryos by day-3 }\end{array}$ & $\begin{array}{l}\text { Day-3 embryos } \\
\text { transferred }\end{array}$ & $\begin{array}{l}\text { Pregnancy } \\
\text { outcome }\end{array}$ \\
\hline Case 1 & $13(11)$ & $5(45)$ & 3 & 8 cell, 7 cell & Singleton Delivered \\
Case 2 & $16(11)$ & $5(45)$ & 2 & 8 cell, 6 cell & Singleton Delivered \\
Case 3 & $14(13)$ & $9(69)$ & 7 & 10 cell, 8 cell & Singleton Delivered \\
& $17(16)$ & $7(43)$ & 2 & 10 cell, 8 cell & Twins Delivered \\
Case 4 & $5(3)$ & $0(0)$ & NA & 7 cell, 6 cell & NA \\
& $3(3)$ & $2(67)$ & 2 & & Terminated Ectopic \\
\hline
\end{tabular}

couple was counseled to consider an attempt using a fresh testicular sperm extraction at the time of oocyte retrieval. The couple chose to pursue this option; eleven mature oocytes were retrieved, all of which were injected with fresh testicular sperm. Five oocytes fertilized with two day three embryos (8 cell, 7 cell) transferred. A resulting healthy singleton pregnancy was delivered.

\section{Case 2}

The couple presented with one failed IVF-ICSI cycle marked by poor embryo development in the context of cryptozoospermia following vasectomy reversal. The female partner was 37 years old with no prior history of conception. Her evaluation was notable only for a small endometrial polyp that was resected. Follow-up transvaginal ultrasound confirmed a normal uterine cavity.

Her partner was a 49-year-old nonsmoker with one prior spontaneous conception with another partner prior to his vasectomy 12 years earlier. He had undergone a vasectomy reversal 4 years after initial vasectomy. On exam his testes were $27 \mathrm{cc}$ in volume, and there were no palpable varicoceles. Although he initially had return of motile sperm to the ejaculate $(1-8$ million total motile sperm per sample), his semen analyses demonstrated a progressive deterioration of motile counts prior to referral to our center. Five preoperative semen analyses were available. At the time of treatment samples required centrifugation with fewer than 20 immotile sperm obtained per pelleted sample. His hormonal profile was otherwise reassuring with an $\mathrm{FSH}$ of $2.0 \mathrm{mIU} / \mathrm{mL}$ and a morning serum testosterone of $423 \mathrm{ng} / \mathrm{dL}$.

The couple's initial IVF-ICSI attempt utilized rare immotile sperm from an ejaculated sample identified only after several hours of searching by two embryologists. A total of five mature oocytes were retrieved and two fertilized. Both embryos arrested at the 2-cell stage prior to a transfer attempt.

Due to the degree of cryptozoospermia and poor embryo development evident with the first cycle attempt, the couple opted to pursue a second cycle using freshlyretrieved testicular sperm. Twitching sperm were obtained and five of 11 mature oocytes fertilized normally.
Two high quality embryos (8-cell, 6-cell) were transferred on day three. A singleton pregnancy resulted with successful term delivery of a healthy male infant.

\section{Case 3}

The third couple was characterized by multiple failed IVF-ICSI cycles using fresh ejaculated immotile sperm in the setting of known Kartagener's Syndrome in the male. The female partner was 32 years old with no prior conceptions or attempts. She had a history of symptomatic mural fibroids for which she underwent laparoscopic myomectomy without complication. Subsequent office hysteroscopy confirmed a normal uterine cavity. Her testing and evaluation were otherwise unremarkable.

Her partner was a 32-year-old nonsmoker who was diagnosed with Kartagener's Syndrome at the age of three. On exam his testes were symmetric and 32 cc's in volume, and there were no clinical varicoceles. His endocrine workup revealed an FSH of $4.9 \mathrm{mIU} / \mathrm{mL}$ and a morning serum testosterone of $437 \mathrm{ng} / \mathrm{dL}$. Semen analyses, as expected, demonstrated a complete absence of motility. Over the course of his evaluation, six semen analyses were performed. Concentrations ranged from 8 to 19 million/cc (total count 16 to 38 million) with 0 to $2 \%$ normal forms by strict criteria.

Following the female partner's myomectomy and post-operative recovery, the couple had undergone three IVF-ICSI attempts using fresh ejaculated sperm. Each cycle resulted in the retrieval of 4 to 12 mature oocytes, with resulting fertilization rates of 0 to $42 \%$. A single blastocyst was transferred during the first cycle without success. The second and third cycles utilized sperm selected based upon the hypo-osmotic swelling assessment in an effort to optimize outcomes. Viability testing was also judged with the mechanical touch technique, which uses a combination of morphology and tail flexibility for assessment [14]. Two day three embryos were transferred with the third cycle resulting in a biochemical pregnancy that did not progress.

Following the three failed cycles, the couple elected for another attempt using fresh testicular sperm. This 
cycle resulted in the retrieval of 13 mature oocytes, nine of which fertilized. Two high quality embryos (10-cell, 8 -cell) were transferred on day three. The couple successfully delivered a singleton pregnancy at term. A single resulting blastocyst was also frozen.

The couple desired further family building and pursued transfer of their remaining frozen blastocyst. Unfortunately no pregnancy resulted, and the couple proceeded to another IVF-ICSI attempt using freshly procured testicular sperm. With this cycle, seven of 16 mature oocytes fertilized normally and two day three embryos were transferred (8-cell, 10-cell). A resulting twin pregnancy was successfully delivered.

\section{Case 4}

The final couple presented with both decreased ovarian reserve and OAT in combination with an elevated sperm DFI. The woman was 38 years old when she initiated treatment at our center. A previous fertility evaluation had demonstrated a day three FSH of 10.8 (normal $<9$ ) and an antral follicle count of 9 (ideally $>14$ ). The remainder of her workup was unremarkable.

Her partner was a 46-year-old nonsmoker who had a history of vasectomy 15 years prior, which was then reversed after a 14 year obstructive interval. He initially had return of motile sperm to the ejaculate with progression over time to OAT. His sperm concentrations dropped from a high of 9 to 0.3 million/cc, presumably due to partial anastomotic stenosis. At the time of our evaluation, by now his fifth semen analysis, sperm concentration had further declined to $0.1 \mathrm{million} / \mathrm{cc}$ with $25 \%$ progressive motility and $3 \%$ normal morphology $(<1$ million total motile sperm). The patient otherwise had a reassuring hormonal profile, with an FSH of 3.8 $\mathrm{mIU} / \mathrm{mL}$ and a morning serum testosterone of $474 \mathrm{ng} / \mathrm{dL}$. His exam demonstrated $30 \mathrm{cc}$ testes bilaterally with a small left varicocele.

The couple's initial IVF-ICSI attempt using fresh ejaculated sperm resulted in the retrieval of 6 mature oocytes, only one of which fertilized. The resulting 5-cell embryo was transferred on day three and ended in biochemical pregnancy without progression. An SCSA was subsequently obtained demonstrating an elevated DFI of $42.8 \%$.

Given the elevated DFI, poor fertilization rate, and unsuccessful outcome of their first cycle, the couple chose to proceed with another IVF-ICSI cycle using fresh testicular sperm. Two subsequent testicular sperm extraction cycles were conducted: the first IVF cycle resulted in the retrieval of three mature oocytes, none of which fertilized following injection with motile sperm. With the second cycle a total of three mature oocytes were retrieved, two of which fertilized. Both embryos were transferred on day three (6-cell, 7-cell). An ectopic pregnancy resulted and was managed medically. Afterward, the couple elected to stop further IVF attempts.

\section{Discussion}

The sperm-related factors that result in limited fertilization, poor embryo development, impaired implantation, and early spontaneous abortion are poorly understood. Historically, sperm motility and morphology have been the primary surrogates for sperm quality; early studies substantiated this concept in terms of poorer male factor-related outcomes using IVF with conventional insemination [15-17]. However, the predictive values of morphology and motility have been questioned when the standard insemination process is bypassed $[18,19]$. In an effort to better characterize sperm quality in the era of IVF-ICSI, investigations into more sophisticated measures of sperm quality such as oxidative damage and impaired DNA integrity have been pursued.

A relationship between oxidative stress and subfertility has now been established. Elevated seminal ROS are negatively correlated with sperm concentration, motility, and morphology [20, 21]. Additionally, elevated ROS are found in men who have undergone vasectomy reversal [22]. Cases 2 and 4 are characterized by OAT in men who were previously treated with vasovasostomy. It is unclear if elevated ROS played a dominant role in these cases, although their presentations can be considered consistent with oxidative stress.

A direct link between sperm oxidative damage and embryo demise has been demonstrated in animal models [23]. Additionally, elevated seminal ROS are correlated with poor embryo development in humans [24]. Relatively little is known regarding the accumulation of oxidative damage as sperm progress through the male reproductive tract. Ollero et al. postulated that the presence of immature spermatozoa, a known source for endogenous ROS [25], may result in damage to mature sperm during transit through the epididymis [26]. The local environment within the epididymis may be further stressed as a vasovasostomy site begins to stenose. As pressure builds within the testicular side of the anastomosis, progressive epididymal dysfunction may ensue, particularly in a system that has suffered iatrogenic obstruction in the past [27]. The use of testicular sperm may bypass this potentially toxic local environment, and may translate into improved IVF-ICSI outcomes in patients with similar profiles as Cases 2 and 4.

A known result of oxidative stress is DNA damage, which is a prominent feature of Cases 1 and 4. For both patients an SCSA revealed a DFI above $40 \%$. In one of the largest series by Bungum et al., a DFI greater than $30 \%$ was found to translate into an odds ratio of 0.1 (CI 0.02 - 0.42) for clinical pregnancy rates following intrauterine insemination [28]. Their data found no 
statistical difference for their conventional IVF and IVF-ICSI cohorts, which was initially attributed to selection bias regarding oocyte quality. A subsequent metaanalysis corroborated these findings regarding IVF-ICSI and an elevated DFI, although a trend toward a higher miscarriage rate was noted [29]. Despite the fact that IVFICSI outcomes remain favorable even in states of marked DNA damage [30], conceivably due to the DNA repair capacity of high quality oocytes [31], the possibility remains that a subset of patients may be particularly affected by an elevated DFI. Greco and colleagues examined a small cohort of couples who were similar to Case 1: patients who were characterized by an elevated DFI and recurrent IVF-ICSI failure using ejaculated sperm [11]. They found that testis-derived samples had significantly less DNA damage. Although fertilization rates and embryo development were no different in the testis versus ejaculated samples, the testicular sperm achieved eight clinical pregnancies as opposed to one in the control group. They postulated that significant DNA damage accrues during transit though the epididymis, similar to the concept raised by Ollero et al. [26].

Some investigators have attempted to treat impaired DNA integrity with antioxidants in order to limit oxidative stress. Abad et al. demonstrated a modest improvement in semen parameters and DFI following 3 months of oral antioxidant therapy in a group of infertile men [32]. In the case of testicular derived sperm, a small case series by Greco et al. found improved ICSI outcomes when men with poor DNA integrity are pretreated with oral antioxidants [33]. A summative review by Kumalic and Pinter substantiated the beneficial effects of antioxidant therapy, albiet most of these results are based upon ejaculated samples [34]. In our practice we routinely offer men with asthenospermia supplementation with coenzyme Q10. It remains unclear if the outcomes would have differed if the men in this case series were offered a formal course of antioxidant therapy. Further studies are needed to expand upon the preliminary results of Greco et al., in which testicular sperm may be further optimized through oral antioxidants [33].

In regards to Case 3, little is known about the pathophysiology of poor IVF-ICSI outcomes in Kartagener's Syndrome. Given the rarity of the disease, most of the primary literature is based upon case reports [35]. It is assumed that Kartagener's Syndrome results in longer transit times through the epididymis, which may result in greater oxidative damage, cell senescence, and loss of DNA integrity [36, 37]. An analysis by Cayan et al. demonstrated substantial improvement of sperm viability with testicular sperm in one patient, presumably bypassing the damage that may develop within the epididymis [38]. Additionally, Westlander and colleagues concluded that more reliable fertilization rates can be achieved with testicular sperm [37]. Future studies are needed to define a link between this rare syndrome and elevated oxidative stress and/or increased DNA fragmentation.

The optimal source of sperm for IVF-ICSI remains controversial [11,39]. Two recent series have compared the results of IVF-ICSI between ejaculated and testicular sperm. Lu et al. found a slight advantage of ejaculated sperm in terms of fertilization rates and embryo quality within one center's large experience [4]. Similarly, Gnoth and colleagues analyzed 337 IVF-ICSI cycles and found no difference in clinical outcomes between ejaculated and testicular sperm for men with cryptozoospermia [6]. Both studies were limited by a retrospective design with significant potential for selection bias. In each case a testicular sperm extraction was used only when sperm could not be reliably found within the ejaculate. Neither study compared IVF-ICSI outcomes between ejaculated and matched testicular derived sperm from the same individual. Additionally, these studies did not stratify for the cohort of men who have had repeated IVF-ICSI failure, a group that may benefit from testis-derived sperm $[10,11,40,41]$. Our small case series documents a possible benefit for this population. It should be noted, however, that our study is also limited by a retrospective design. Additionally, the criteria used to select for PercBx was not standardized at our institution, and so one must consider a component of selection bias. Despite these limitations in study design, our data do suggest an indication for retrieving testicular sperm despite sperm availability within the ejaculate. Further studies are needed to confirm our observations.

\section{Conclusions}

It remains to be elucidated if patients with recurrent IVF-ICSI failure have progressive deterioration of sperm quality, and therefore worse pregnancy outcomes, due to sperm passage through the male reproductive tract [26]. Larger studies are ultimately needed to substantiate these pilot investigations and preliminary observations. Until these data are obtained, for couples who undergo repeated unsuccessful cycles of IVF-ICSI with ejaculated sperm, a trial of freshly procured testicular sperm may offer an improved chance to achieve parenthood.

\section{Ethics approval}

This study was approved by the Massachusetts General Hospital Institutional Review Board.

\section{Consent for publication}

Not applicable

\section{Availability of data and material}

All data leading to the conclusions of this paper are available in the results section of this manuscript. 


\section{Abbreviations}

DFI: DNA fragmentation index; FSH: follicle stimulating hormone; IVF: in vitro fertilization with conventional insemination; IVF-ICSI: in vitro fertilization and intracytoplasmic sperm injection; OAT: oligoasthenoteratospermia; PercBx: percutaneous testis biopsy; ROS: reactive oxygen species; SCSA: sperm chromatin structure assay.

\section{Competing interests}

The authors declare that they have no competing interests.

\section{Authors' contributions}

$\mathrm{RH}$ - data analysis, data interpretation, manuscript generation. DW - data collection, study design, critical review of manuscript. $\Pi$-study design, critical review of manuscript. CT - data collection, study design, data interpretation, manuscript generation. All authors read and approved the final manuscript

\section{Acknowledgements}

None

\section{Funding}

None

\section{Author details}

${ }^{1}$ Department of Urology, Massachusetts General Hospital, Boston, MA, USA. ${ }^{2}$ Inception Infertility, Houston, TX, USA. ${ }^{3}$ Department of Obstetrics \& Gynecology, Massachusetts General Hospital, Boston, MA, USA. ${ }^{4}$ MGH Fertility Center, 55 Fruit Street, YAW 10A, Boston, MA 02114, USA

\section{Received: 2 February 2016 Accepted: 22 March 2016}

\section{Published online: 15 April 2016}

\section{References}

1. Lande Y, Seidman DS, Maman E, Baum M, Dor J, Hourvitz A. Couples offered free assisted reproduction treatment have a very high chance of achieving a live birth within 4 years. Fertil Steril. 2011:95:568-72.

2. Olivius C, Friden B, Borg G, Bergh C. Why do couples discontinue in vitro fertilization treatment? A cohort study. Fertil Steril. 2004;81:258-61.

3. Luke B, Brown MB, Wantman E, Lederman A, Gibbons W, Schattman GL, Lobo RA, Leach RE, Stern JE. Cumulative birth rates with linked assisted reproductive technology cycles. N Engl J Med. 2012;366:2483-91.

4. Lu Y, Gao H, Li B, Zheng Y, Ye Y, Qian Y, Xu C, Huang H, Jin F. Different sperm sources and parameters can influence intracytoplasmic sperm injection outcomes before embryo implantation. J Zhejiang Univ Sci B. 2012;13:1-10.

5. Nagy ZP, Liu J, Joris H, Verheyen G, Tournaye H, Camus M, Derde MC, Devroey $P$, Van Steirteghem AC. The result of intracytoplasmic sperm injection is not related to any of the three basic sperm parameters. Hum Reprod. 1995:10:1123-9.

6. Gnoth C, Markhinin V, Maxrath B, Skonieczny T, Friol K, Roos J, Rahimi G, Godehardt E. Impact of sperm cell source on the results of intracytoplasmic sperm injection. Arch Gynecol Obstet. 2014;291:663-9.

7. Guo Y, Dong R, Su Y, Li J, Zhang Y, Sun Y. Follow-up of children born after intracytoplasmic sperm injection with epididymal and testicular spermatozoa. Chin Med J (Engl). 2013;126:2129-33.

8. Fedder J, Loft A, Parner ET, Rasmussen S, Pinborg A. Neonatal outcome and congenital malformations in children born after ICSI with testicular or epididymal sperm: a controlled national cohort study. Hum Reprod. 2013;28:230-40.

9. Negri L, Patrizio P, Albani E, Morenghi E, Benaglia R, Desgro M, Levi Setti PE. ICSI outcome is significantly better with testicular spermatozoa in patients with necrozoospermia: a retrospective study. Gynecol Endocrinol. 2014;30:48-52.

10. Ben-Ami I, Raziel A, Strassburger D, Komarovsky D, Ron-El R, Friedler S. Intracytoplasmic sperm injection outcome of ejaculated versus extracted testicular spermatozoa in cryptozoospermic men. Fertil Steril. 2013;99:1867-71.

11. Greco E, Scarselli F, lacobelli M, Rienzi L, Ubaldi F, Ferrero S, Franco G, Anniballo N, Mendoza C, Tesarik J. Efficient treatment of infertility due to sperm DNA damage by ICSI with testicular spermatozoa. Hum Reprod. 2005;20:226-30.
12. Suganuma $R$, Yanagimachi $R$, Meistrich ML. Decline in fertility of mouse sperm with abnormal chromatin during epididymal passage as revealed by ICSI. Hum Reprod. 2005;20:3101-8.

13. Hauser R, Bibi G, Yogev L, Carmon A, Azem F, Botchan A, Yavetz H, Klieman SE, Lehavi O, Amit A, Ben-Yosef D. Virtual Azoospermia and Cryptozoospermia-Fresh/Frozen Testicular or Ejaculate Sperm for Better IVF Outcome? J Androl. 2011;32:484-90.

14. Marques de Oliveira N, Sánchez RV, Rodriguez Fiesta S, Lopez Salgado T, Rodríquez R, Bethencourt JCA, Zamora RB. Pregnancy with frozen-thawed and fresh testicular biopsy after motile immotile sperm microinjection, using the mechanical touch technique to assess viability. Hum Reprod. 2004; 19:262-5.

15. Grow DR, Oehninger S, Seltman HJ, Toner JP, Swanson RJ, Kruger TF, Muasher SJ. Sperm morphology as diagnosed by strict criteria: probing the impact of teratozoospermia on fertilization rate and pregnancy outcome in a large in vitro fertilization population. Fertil Steril. 1994;62:559-67.

16. Kruger TF, Acosta AA, Simmons KF, Swanson RJ, Matta JF, Oehninger S. Predictive value of abnormal sperm morphology in in vitro fertilization. Fertil Steril. 1988:49:112-7.

17. Nagy ZP, Verheyen G, Tournaye H, Van Steirteghem AC. Special applications of intracytoplasmic sperm injection: the influence of sperm count, motility, morphology, source and sperm antibody on the outcome of ICSI. Hum Reprod. 1998;13 Suppl 1:143-54.

18. Hershko-Klement A, Rovner E, Yekutieli D, Ghetler Y, Gonen O, Cohen I, Wiser A, Berkovitz A, Shulman A. Embryo quality and implantation rates are not influenced by total motile count values in an ICSI programme: A novel point of view. Int J Mol Epidemiol Genet. 2012;3:205-12.

19. McKenzie LJ, Kovanci E, Amato P, Cisneros P, Lamb D, Carson SA. Pregnancy outcome of in vitro fertilization/intracytoplasmic sperm injection with profound teratospermia. Fertil Steril. 2004;82:847-9.

20. Agarwal A, Tvrda E, Sharma R. Relationship amongst teratozoospermia, seminal oxidative stress and male infertility. Reprod Biol Endocrinol. 2014;12:45.

21. Kao SH, Chao HT, Chen HW, Hwang TIS, Liao TL, Wei YH. Increase of oxidative stress in human sperm with lower motility. Fertil Steril. 2008;89:1 183-90.

22. Nandipati KC, Pasqualotto FF, Thomas AJ, Agarwal A. Relationship of interleukin- 6 with semen characteristics and oxidative stress in vasectomy reversal patients. Andrologia. 2005;37:131-4.

23. Burruel V, Klooster KL, Chitwood J, Ross PJ, Meyers SA. Oxidative damage to rhesus macaque spermatozoa results in mitotic arrest and transcript abundance changes in early embryos. Biol Reprod. 2013:89:72.

24. Zorn B, Vidmar G, Meden-Vrtovec $H$. Seminal reactive oxygen species as predictors of fertilization, embryo quality and pregnancy rates after conventional in vitro fertilization and intracytoplasmic sperm injection. Int J Androl. 2003;26:279-85.

25. Agarwal A, Virk G, Ong C, du Plessis SS. Effect of oxidative stress on male reproduction. World J Mens Health. 2014;32:1-17.

26. Ollero M, Gil-Guzman E, Lopez MC, Sharma RK, Agarwal A, Larson K, Evenson D, Thomas AJ, Alvarez JG. Characterization of subsets of human spermatozoa at different stages of maturation: implications in the diagnosis and treatment of male infertility. Hum Reprod. 2001;16:1912-21.

27. Schiff J, Chan P, Li PS, Finkelberg S, Goldstein M. Outcome and late failures compared in 4 techniques of microsurgical vasoepididymostomy in 153 consecutive men. J Urol. 2005:174:651-5. quiz 801.

28. Bungum M, Humaidan P, Axmon A, Spano M, Bungum L, Erenpreiss J, Giwercman A. Sperm DNA integrity assessment in prediction of assisted reproduction technology outcome. Hum Reprod. 2007;22:174-9.

29. Zini A, Sigman M. Are tests of sperm DNA damage clinically useful? Pros and cons. J Androl. 2009;30:219-29.

30. Dar S, Grover SA, Moskovtsev SI, Swanson S, Baratz A, Librach CL. In vitro fertilization-intracytoplasmic sperm injection outcome in patients with a markedly high DNA fragmentation index (>50 \%). Fertil Steril. 2013;100:75-80.

31. Marchetti F, Essers J, Kanaar R, Wyrobek AJ. Disruption of maternal DNA repair increases sperm-derived chromosomal aberrations. Proc Natl Acad Sci U S A. 2007:104:17725-9.

32. Abad C, Amengual MJ, Gosálvez J, Coward K, Hannaoui N, Benet J, GarcíaPeiró A, Prats J. Effects of oral antioxidant treatment upon the dynamics of human sperm DNA fragmentation and subpopulations of sperm with highly degraded DNA. Andrologia. 2013:45:211-6.

33. Greco E, Romano S, lacobelli M, Ferrero S, Baroni E, Minasi MG, Ubaldi F, Rienzi L, Tesarik J. ICSI in cases of sperm DNA damage: beneficial effect of oral antioxidant treatment. Hum Reprod. 2005;20:2590-4. 
34. Imamovic Kumalic S, Pinter B. Review of clinical trials on effects of oral antioxidants on basic semen and other parameters in idiopathic oligoasthenoteratozoospermia. Biomed Res Int. 2014;2014:426951.

35. Dávila Garza SA, Patrizio P. Reproductive outcomes in patients with male infertility because of Klinefelter's syndrome, Kartagener's syndrome, roundhead sperm, dysplasia fibrous sheath, and "stump" tail sperm: an updated literature review. Curr Opin Obstet Gynecol. 2013;25:229-46.

36. Nuñez R, López-Fernández C, Arroyo F, Caballero P, Gosálvez J. Characterization of sperm DNA damage in Kartagener's syndrome with recurrent fertilization failure: case revisited. Sex Reprod Healthc. 2010;1:73-5.

37. Westlander G, Barry M, Petrucco O, Norman R. Different fertilization rates between immotile testicular spermatozoa and immotile ejaculated spermatozoa for ICSI in men Kartagener's syndrome: case report. Hum Reprod. 2003;18:1286-8.

38. Cayan S, Conaghan J, Schriock ED, Ryan IP, Black LD, Turek PJ. Birth after intracytoplasmic sperm injection with use of testicular sperm from men with kartagener/immotile cilia syndrome. Fertil Steril. 2001;76:612-4.

39. Amirjannati N, Heidari-Vala H, Akhondi MA, Hosseini Jadda SH, Kamali K, Sadeghi MR. Comparison of intracytoplasmic sperm injection outcomes between spermatozoa retrieved from testicular biopsy and from ejaculation in cryptozoospermic men. Andrologia. 2012;44:704-9.

40. Bendikson KA, Neri QV, Takeuchi T, Toschi M, Schlegel PN, Rosenwaks Z Palermo GD. The outcome of intracytoplasmic sperm injection using occasional spermatozoa in the ejaculate of men with spermatogenic failure. J Urol. 2008;180:1060-4.

41. Weissman A, Horowitz E, Ravhon A, Nahum H, Golan A, Levran D. Pregnancies and live births following ICSI with testicular spermatozoa after repeated implantation failure using ejaculated spermatozoa. Reprod Biomed Online. 2008;17:605-9.

\section{Submit your next manuscript to BioMed Central and we will help you at every step:}

- We accept pre-submission inquiries

- Our selector tool helps you to find the most relevant journal

- We provide round the clock customer support

- Convenient online submission

- Thorough peer review

- Inclusion in PubMed and all major indexing services

- Maximum visibility for your research

Submit your manuscript at www.biomedcentral.com/submit

) Biomed Central 